\title{
Opposition to Vaccination and Immunisation the UK Experience - from Smallpox to MMR
}

\section{David Baxter}

Clinical Epidemiology and Public Health Unit, School of Translational Medicine, Manchester University Medical School, Manchester, UK

*Corresponding author: David Baxter, Clinical Epidemiology and Public Health Unit, School of Translational Medicine, Manchester University Medical School, Manchester, UK, Tel: 44161306 0460; E-mail: baxter@nhs.net

Received date: 06 Aug 2014; Accepted date: 18 Sep 2014; Published date: 25 Sep 2014

Copyright: (C) 2014 Baxter D, et al. This is an open-access article distributed under the terms of the Creative Commons Attribution License, which permits unrestricted use, distribution, and reproduction in any medium, provided the original author and source are credited

\begin{abstract}
Immunisation is a key public health intervention with protection from direct and/or indirect (herd) immunity: this is directly related to vaccine uptake rates, and anything therefore that interferes with uptake, including opposition to vaccination by individuals, has potentially serious consequences for the control of specific vaccine preventable infections (VPIs). The UK has a history of opposition by minority groups to smallpox, whooping cough and measles, mumps and rubella (MMR) vaccination, which this paper will review, as it is believed that an understanding of their causes has implications for any VPI disease control through current and future universal immunisation programmes.
\end{abstract}

Keywords: Immunisation; Vaccination; Vaccines; Immunity

\section{Introduction}

Immunisation is a key public health intervention with protection from direct and/or indirect (herd) immunity: this is directly related to vaccine uptake rates, and anything therefore that interferes with uptake, including opposition to vaccination by individuals, has potentially serious consequences for the control of specific vaccine preventable infections (VPIs). During the last two hundred years there have been several vaccine controversies in the UK where opposition by community groups impacted on programme delivery. This paper will consider the three most important and serious namely smallpox, whooping cough and measles, mumps and rubella (MMR) vaccination, as it is believed that an understanding of their causes and subsequent progression may provide valuable assistance when responding to any future incident.

\section{The First Controversy - Smallpox}

Smallpox immunisation in the UK almost certainly started in the late $16^{\text {th }}$ or early $17^{\text {th }}$ century, although it had been used in other countries for many hundreds of years [1,2]. Inoculation (also known as variolation, incision or ingrafting) to protect against smallpox was first reported to the Royal Society in 1714 by Emmanuel Timonius [3]. Subsequently in 1722, Lady Mary Wortley Montagu together with several members the College of Physicians conducted the first reported vaccine trial with six inmates of Newgate Prison being offered their freedom if they underwent this same procedure of having wild smallpox material introduced into their skin by scarification $[4,5]$.

The successful outcome of this trial, together with the uptake of inoculation by key individuals including the Royal Family and its promotion by leading medical figures led to increased uptake of inoculation [6]. However, this was only gradual with "opposers" of inoculation being drawn from four main sources.

First, opposition came from both the religious and scientific communities based on their differing views of symptom causation - the twin concepts of disease and pathology not being part of contemporary thought in the $18^{\text {th }}$ and early $19^{\text {th }}$ century [6]. There were a number of theories about why a person became ill: these were often contradictory, but the prevailing theories relevant to inoculation were that illness was the result of divine retribution for being a sinner, an imbalance of the body humours - blood, phlegm, black bile and yellow bile (the humoral theory), exposure to vapours (miasmas) coming from putrefying material, the Methodist theory (where disease was due to an imbalance of two opposing tendencies for example too little tone, sthenic or too much tone asthenic), or exposure to a specific unspecified entity (the contagion theory) [7].

Given the diversity of views on symptom causation, it is unsurprising that treatments were similarly varied. Interfering with God's plans by inoculation was morally wrong for those who believed that disease and suffering were sent in the wisdom and goodness of divine Dispensations: or put rather more simply "Let us do evil, that good may come" $[8,9]$. The humoral theory regarded illness symptoms as arising from an imbalance between the four body humors with appropriate treatments being their rebalance, for example by blood letting. Inoculation did not fit with the miasma theory either whose supporters argued for hygiene measures (for example community sanitary measures) to reduce exposure - they also stressed the importance of living a moral life because this theory also regarded illnesses as modifiable by host factors. For "Methodists" if excessive tone was the cause of disease, then treatment was with opium, and alcohol for those diseases caused by lack of tone. Inoculation didn't fit with the contagion theory because no specific entities (i.e. pathogens) had been identified - this being nearly a hundred years before the Germ theory became accepted [7,10-12].

Second was the initial reluctance based on safety concerns although both Timonius and Montagu reported that the procedure was entirely safe [13], reports soon emerged in the popular press of the occurrence of smallpox shortly after inoculation in both recipients and close contacts, with occasional deaths: in addition other diseases (for example syphilis) were believed to be transmitted by the procedure [14-16]. These were real fears - in 1726 a comparative analysis was published that reported a mortality rate of 13 out of 624 (one in 48 ) for 
individuals being inoculated compared with an expected mortality of one in six had they not undergone the procedure but developed natural smallpox - the author concluded that "inoculation may reasonably be reckoned to have saved 91 lives out of 104 [in those inoculated] that would otherwise have been lost, and might have saved 2,491 out of 2,848 which actually perishes by this distemper" [17]. The decision to be inoculated therefore required careful consideration unless wild smallpox was circulating in the community.

Third was the view that the procedure was ineffective and didn't actually prevent smallpox. This arose from the belief that the development of modified smallpox post inoculation was necessary for protection against wild disease and if this did not occur then the procedure had not worked. Success or failure of the procedure could often be found in the popular press - thus an article in 1724 reported that "it is much to be feared that the inoculation project upon the Duke of Bedford will not have its desired effect, the smallpox not appearing thereupon, but the Lady Betty his sister, has that distemper come out upon her in a very good kind" [18].

And finally there was resistance to vaccination from various "medical" practitioners. With many thousands of cases and as many as $20 \%$ of all deaths annually being due to smallpox, demand for treatment was considerable. In the early $18^{\text {th }}$ century there were various groups who would provide this care including Physicians, Surgeons, and Apothecaries - Quacks and other amateur practitioners also provided a service to all members of society. Once smallpox had developed, the various treatments commonly prescribed reflected the belief systems of both the affected person and the practitioner - the individualistic nature of symptoms meant there was an endless array of ailments patients might experience and cures they might be prescribed - these included plant remedies and potions, the application of machines (for example the stomach brush), and physical procedures (generally purging, bleeding, sweating and the administration of alcohol with spirit of vitriol): isolation, quarantining and limewashing of the homes where infected people had lived were also advocated with varying degrees of success depending on how consistently these were carried out $[19,20]$. These practitioners thus had a vested interest in resisting the introduction of inoculation, as this could affect their livelihood.

Inoculation was initially opposed by Physicians, the "medical elite" of the time, but once it became favoured and indeed fashionable among the upper classes they promoted it - often making an initially simple procedure more complex (and expensive), and hence not suitable for other practitioners, for example by insisting on the need for fasting and bleeding prior to it $[6,21]$. In contrast at least one surgeon, John Birch opposed inoculation because the vaccine was too effective and this prevented smallpox being an "important method of decreasing the population particularly in large and poor families" [14].

In the late $18^{\text {th }}$ century immunisation with cowpox virus (i.e. vaccination) started to replace inoculation as a protection against smallpox. Although not the first person in England to use vaccination, Jenner provided the evidence-base for this change and indeed popularised the procedure. In part, Jenner's interest in smallpox may have arisen from being variolated as an eight year old child when he underwent bleeding and purging in the six weeks prior to the procedure following which he was confined until he had recovered. It is reported that he subsequently developed long-term (unspecified) health problems as a consequence, although the evidence for this is conflicting $[16,22]$. Certainly as a physician Jenner inoculated his own patients and would have been aware of the adverse events attendant on the procedure. These risks, including an estimated mortality rate that might be as high as $2 \%$ had considerably limited uptake even during smallpox outbreaks and the more general acceptance of Jenner's vaccine was a result of its perceived enhanced safety profile compared with inoculation [14,23].

Vaccination spread widely in the early 1800 s particularly internationally - in the UK by contrast uptake was lower - in 1825 it was estimated that only half the children born in large towns were being vaccinated with much lower figures $(\sim 5-15 \%)$ being reported elsewhere [14,24]. Opposition to vaccination, as with inoculation predictably arose for the reasons identified above - namely contrary views on disease causation, safety and efficacy concerns about vaccination, and opposition from practitioners of inoculation. The influence of the miasma theory continued in the promotion of hygiene and sanitary measures, together with a good state of personal health by antivaccine supporters [2].

As vaccine uptake rates were insufficient to prevent disease transmission, outbreaks of smallpox thus continued and in order to better control disease Parliament introduced an "Act to Extend the Spread of Vaccination" in July, 1840 with the twin aims of both banning inoculation (upon penalty of one month imprisonment) and promoting vaccination by requiring the Poor law Commissioners to provide vaccination services for all the community, rather than only the "destitute" as previously: medical officers were paid $1 \mathrm{~s}$ and $6 \mathrm{p}$ per vaccination $[2,25]$. The issue of cost, which must have been a deterrent for some since most healthcare was largely privately provided, was thus addressed by making vaccination free - this reflected a view, not previously acknowledged, that Parliament had a direct responsibility for the healthcare of its citizens.

Despite this legislation vaccination rates remained suboptimal and smallpox continued to circulate with 16,000 deaths in the decade 1840-1850 for an estimated death rate of about 305 per million [14,24]. The failure to take up vaccine was largely attributed to "indifference and neglect, for as soon as the smallpox broke out in a district, people flocked to be vaccinated without caring whether the matter was from animals or not" [24]. To address this, Parliament passed the Vaccination Extension Bill in 1853, which introduced compulsory vaccination for all unvaccinated people including migrants and for all infants within 3 months of birth - parents who didn't comply with the legislation were liable for a fine and/ or imprisonment. In 1867 compulsory vaccination was extended to children up to 14 years of age with cumulative penalties for defaulters $[2,26]$.

This legislation reflected a view among some parts of society, including the governing elite, that the State had a legitimate right to require its citizens to be medically treated (in this case by vaccination) to prevent contagious disease spread among the remainder of the population. Opposition to both variolation and vaccination which had been low level and uncoordinated now focused on the compulsory nature of the Act and came immediately from those who viewed the Act as an infringement of civil liberties, with the Anti-Vaccination League (formed in 1853) providing one means of co-ordinating these activities, including public disobedience [26,27]. The aim of the antivacccine lobby was to overturn the compulsory element of the legislation with many also wanting to discredit vaccination in favour of the implementation of sanitary measures. A number of supporters chose to go to prison rather than accede to the Act $[28,29]$.

The issues raised by compulsory vaccination were addressed in newspapers, pamphlets, and cartoons, which were the principal means 
of mass communication (as opposed to the telegraph and telephone for the individual) in the mid $19^{\text {th }}$ century. The nature of these different communication pathways, however, meant that it took some considerable time for ideas to be disseminated, and furthermore having to write them down gave the opportunity to consider them in detail as opposed to the more instant nature of speech. One common antivaccine argument was that if vaccination is effective, then the only danger is to unvaccinated people, who have made that decision for themselves, or in the case of minors by their parents or guardians [30]. Countering this was a challenge because although vaccination was evidently highly effective - in its absence smallpox caused considerable mortality and morbidity - it didn't always protect as was clear from the occurrence of smallpox in some who had been vaccinated; furthermore it occasionally caused serious adverse events, including death among vaccine recipients.

This line of argument relates to herd immunity and is role in VPI control, and would appear from the smallpox experience to be largely determined by perceptions of disease infectivity and morbidity/ mortality, vaccine efficacy and side effect profile, and the extent to which other methods provide effective disease control - thus in Leicester for example local government reluctance to enforce compulsory vaccination led to efforts to control disease by the reintroduction of the use of prompt recognition, isolation of cases in hospital and quarantining of contacts in their homes - by no means new this became known as the "Leicester Method" for outbreak control. Quarantine was made compulsory in Leicester in 1879 [31].

Throughout the late $18^{\text {th }}$ and $19^{\text {th }}$ centuries, there were a very small number of physicians and scientists who argued against vaccination some are depicted in a popular cartoon (the Vaccination Monster) of the time [32]. The highly respected scientist Alfred Wallace, involved with Darwin in developing the theory of evolution, wrote of the dangers of vaccination. However, as experience accumulated and the germ theory of disease became more widely accepted, such individuals became more and more peripheral to the debate and isolated [33].

Vaccination rates for infants between 1853 and 1898 were about 80-85\%. Nevertheless, the compulsory nature of the programme was a cause of continuing public disquiet and legislation in 1898 and 1907 introduced a conscience clause allowing objectors to avoid the punitive penalties of not vaccinating their child. The advent of the National Health Service in 1948 saw the end of compulsory vaccination. Over this period infant vaccination rates declined from $63 \%$ in 1907 to $18.2 \%$ in 1948 [14].

\section{The Second Controversy - Whooping Cough}

By the end of the $19^{\text {th }}$ century, the concept of disease and theories of causation were developing that more reflected the application of the scientific method to medical knowledge [7]. The germ theory of disease that had been promulgated in the 1860 s by Pasteur and later by Koch became more generally accepted, with the agents that caused human anthrax, erysipelas, diphtheria, tuberculosis and relapsing fever being discovered in the 1880s [34,35]. Following on from this, host defences against infection began to be studied with the emergence of two competing theories - the cellular and the humoral (or antibody). The former was based on the work of Metchnikoff who had identified phagocytes as the basis for recovery from infection; and the latter on various researchers including Behring and Koch. Of particular note is the antibody theory providing a scientific basis for immunisation against certain infections [14,23].
In rapid succession vaccines against rabies and typhoid were developed, but neither was used universally and opposition to their use was limited [14]. During the $20^{\text {th }}$ century there were two vaccine controversies in the UK where the resultant opposition to the separate programmes had a substantial and continuing impact on both public and professional opinion, and on vaccine uptake. The first concerned whooping cough and the second the MMR vaccines.

In the early to mid $20^{\text {th }}$ century, whooping cough affected more than $60-70 \%$ of infants and children with about 9,000 deaths annually - the highest of any infectious disease in England and Wales at that time: epidemics occurred every $3 / 4$ years. The causative organism, Bordetella pertussis was first isolated in 1906 by Bordet and Gengou who in 1912 also developed killed, whole cell (WC) vaccines for use in children [36]. A more systematic study of a pertussis WC vaccine was carried out in the Faroe Islands in the 1920s by Madsen, and these demonstrated a reduction in mortality among vaccinated individuals compared with non vaccinated controls ( $0.15 \%$ compared with $2.4 \%)$ $[14,37]$. Subsequently in the 1940 s combination vaccines with diphtheria and tetanus toxoids were developed and following successful trials in the US were recommended for primary immunisation of infants there in 1947 [14,36,38].

In the UK initial trials showed no benefits of UK produced pertussis vaccines [39]. However, when Kendrick's US vaccine was used the Medical Research Council (MRC) trials showed it to be effective in reducing disease occurrence: there were evident variations in both efficacy and reactogenicity between the four trial preparations, with for example the subunit Pillemer's antigen vaccine being both the most immunogenic and reactogenic. The results led in 1957 to the national introduction of a combined Diphtheria, WC Pertussis and Tetanus (DPT) vaccine for children, and by 1960 seventy per cent of infants had received the DPT with $70 \%$ of all children being vaccinated by 1969 [14,36,38-41].

Both in trials and routine use, WC pertussis vaccine either monovalent or in combination had commonly caused systemic reactions (including a fever) in the first 24-48 hours post immunisation - in part presumably associated with an innate inflammatory response mediated through the Toll-like receptor 4 agonist Lipopolysaccharide contained in the capsule of the WC vaccine. Some authors had also reported early onset seizures, hypotonic-hyporesponsive like episodes and encephalopathy - post encephalopathy a few children had also reportedly experienced regression or failure of further development [42-44]. It is relevant that in the 1950s British MRC trials of WC pertussis vaccines, children with a personal or family history of cerebral lesions were excluded from participation. Madsen had also drawn attention to potentially serious adverse events when he reported the deaths of two Danish infants 30 minutes and two hours after receiving their second whooping cough vaccine [37].

Post introduction of the universal infant programme, whooping cough incidence declined dramatically: for example dropping from 12.1 per 1000 in 1944 in under 1 year olds to 1.6 per 1000 in $1970-73$ [40]. However, in October 1973, John Wilson, a paediatric neurologist presented a paper on the neurological complications of DPT inoculation in infancy at the British Paediatric Association, in which he related the experience of 36 children seen at the 'Hospital for Sick Children', the majority of whom had developed an encephalopathic illness within one week of receiving DPT. He concluded with a recommendation that the Department of Health's contraindications to the pertussis component of the vaccine should be more rigorously 
applied [45-47]. The case series of 36 children was subsequently published and shortly thereafter Wilson recommended setting up a State compensation scheme for vaccine-damaged children $[48,49]$.

The impact of this paper and the attendant publicity should not be underestimated - on 29/01/1974, Jack Ashley MP asked a series of questions in Parliament about adverse events following vaccination, including the possibility of a vaccine damage scheme [50]. In April 1974, the Independent Television (ITV) company broadcast a programme on whooping cough during which Wilson said that he was convinced about the link between pertussis vaccine and severe illness including fits, unconsciousness and focal neurological signs. On the 25/09/74, a lead editorial in the Daily Mail stated 'we are not told that 80 children every year suffer brain damage because of whooping cough vaccine, and just as many die. We are not told that there are possibly hundreds of teenagers with the body of an adult and the mind of a child because they were vaccinated' [51].

Two other doctors added their voice to the antivaccine debate: Professor Stewart, a Glasgow epidemiologist initially questioned whether the decline in incidence of whooping cough in the UK was due to vaccination or changes in socio-economic status and environmental changes: he also raised doubts about vaccine efficacy [52-54]. In a subsequent paper he reported on adverse vaccine reactions drawing conclusions from a case series of 160 children who had been referred to him - he stated that the pertussis reaction syndrome or subsequent brain damage and mental defect may occur at a frequency between 1 in 60,000 to 1 in 10,000, with a transient form still higher: he suggested that if the risk is 1 in 20,000 then as many as 30 children each year would experience permanent brain damage and may be started on the path of early organic dementia, which in its ultimate form has the features of demyelinating disease and cerebral atrophy [55]. The figure for severe brain damage was later revised to 1 in 50,000 based on a study of 1127 children [56]. Professor Dick, a member of the highly influential Joint Committee on Vaccination and Immunisation (JCVI) which had released a paper supporting the pertussis programme took a dissenting view from other JCVI members and recommended that whooping cough vaccine should be offered only on a targeted basis to high risk, lower social class infants until more data on adverse events and vaccine efficacy were available: he also took a similar line to Stewart and questioned the role of vaccine in controlling whooping cough disease [57].

Opposition from the public came initially from individual parents relating experiences in the media of their own brain-damaged children. About 300 also came to Parliament to support Jack Ashley's speech on 29/01/1974 (see above). Subsequently they organised themselves into the Association of Parents of Vaccine-Damaged Children, a parent advocacy group started by Rosemary Fox (whose own daughter she believed had been damaged by vaccination) and they presented information to the Royal Commission on Civil Liberty and Compensation for Personal Injury that was then investigating phocomelia caused by the administration of Thalidomide in pregnancy. The Association also presented information to the Health Service Ombudsman claiming that they had been given insufficient information at the time of their own children's immunisation programme to give informed consent. The Ombudsman reported that the Department of Health (DH) should indeed have highlighted the information it was providing for parents earlier when it became aware of potential adverse events associated with pertussis vaccine [58].

Clinical Medical Officers (CMOs) and Health Visitors (HVs) who largely administered the immunisation programme in England and
Wales, together with General Practitioners (GPs) found themselves caught in a media maelstrom with adverse publicity regularly reported in the media, but no initial rejection on the putative link between brain damage and pertussis vaccine, from official groups/ organisations other than from the JCVI. Professional concern among CMOs, HVs and GPs was such that a number looked for reasons not to vaccinate with pertussis containing vaccines, rather than vaccinate with a consequent impact on uptake [59].

In response to the adverse publicity and the fall in completed primary courses for whooping cough at two years of age from $77 \%$ in 1972 to $31 \%$ in 1976, the DH set up the National Childhood Encephalopathy Study (NCES) to determine if there was any association between whooping cough vaccine and chronic brain damage. The NCES concluded that pertussis-containing vaccines might on very rare occasions cause an encephalopathy, but that because of the small size of the study the risk was too low to be measured [60-62]. That conclusion of a link was successfully challenged and overturned both in the literature and in court [63-65]. It took, however, an extensive pertussis outbreak in the late 1970s (with more than 60 infant deaths), a switch from a whole cell to an acellular vaccine in the 1980s, the Canadian IMPACT study and nearly 20 years in the UK to restore parental confidence in the pertussis vaccine programme as evidenced by a return to the pre-controversy vaccine uptake rates.

\section{The Third Controversy - Measles, Mumps and Rubella (MMR) Vaccine}

Some 10 years after the whooping cough controversy had largely resolved, another one started following the publication of a Lancet paper in 1998: on February 28th, researchers at the Royal Free Hospital reported a possible association between atypical inflammatory bowel disease and pervasive development disorder (PDD), and administration of MMR vaccine $[66,67]$. Although the paper itself was quite measured in the conclusion that no association was proven between MMR and autism, the lead author suggested at a press conference the following day that single vaccines should be used until further evidence about a link became available.

In March 1998, an MRC expert group reported that there was no evidence of a link between MMR, inflammatory bowel disease and autism. In April 1998, Peltola et al. reported from Finland on their longitudinal study of 3 million children who had received MMR vaccine of whom 31 developed bowel disease within 15 days of vaccination, none of whom on follow up had developed autism [68]. In April 2000 Wakefield and O'Leary (a research collaborator) appeared before a US Congressional hearing to present their research. Wakefield and Montgomery (another collaborator) subsequently published a review in December 2000 that claimed weaknesses in how MMR was tested prior to its introduction, particularly the short (28 days) follow up period in the pre-licensing trials [69].

On the 03/02/2002 the influential BBC programme Panorama broadcast, "MMR: Every Parent's Choice" which looked at how safe was the MMR vaccine. The programme included three children whose families were convinced that their children had developed autism post-MMR. Wakefield appeared and reaffirmed his view that MMR should not be used until a link with autism and bowel disease had been disproved. His research collaborator, O'Leary who had reported finding measles virus in the gastro-intestinal tract of children with autism and new variant inflammatory bowel disease also appeared 
[70]. Several authors and organisations subsequently published data showing no association between MMR and autism [68,71-74].

Wakefield was referred to the General Medical Council (GMC) on professional misconduct charges relating to his research. He appeared before them between July 2007 and May 2010 when he was found guilty of all charges and struck off. The attendant publicity throughout the period of the trial brought in coverage of family's with an autistic child leading to quite biased reporting [75]. Data suggested that the media coverage was quite negative - Hussain et al. for example found that $37 \%$ of immunisation publications in the mass media were negative to immunisation [76]. In Sweden in 2004, Zetterstrom wrote that the "media have also refused to report that no relationship exists between MMR vaccination and autistic spectrum disorders or chronic inflammatory bowel disease" [77].

The impact of this adverse publicity in England and Wales was a decline in uptake of MMR in two year old children from a high of $91.8 \%$ in 1995 to about $80 \%$ in the early 2000 s although parts of the country saw a fall to less than $60 \%$ : it took until 2011 for MMR vaccine uptake to reach $91 \%$ - the pre controversy level.

\section{Conclusions}

The three vaccine controversies described in this paper illustrate how opposition to vaccine arises and spreads. It also describes both the lay and official response to such a controversy - the latter is useful for immunisation service providers (including vaccine manufacturers and regulatory agencies) as it will assist in the management of future incidents.

The smallpox controversy started more than 250 years ago and its origin is lost in antiquity, nevertheless study of what happened identifies the issues that opponents focus on. Thus with both variolation and vaccination, opponents concentrated on the rationale for vaccination, vaccine safety and efficacy and alternative methods of disease control and management. This incident differed from both pertussis and MMR in that the smallpox vaccines controversy also involved opposition to the compulsory component of the programme - indeed this was arguably the focus for mass opposition to vaccination.

Opponents focused on the rationale for smallpox vaccination. At this time disease aetiology theories had not been developed and both health practitioners and lay people were concerned only with symptoms, which they attempted to explain and manage in ways that were dependent on their views of causation. Thus there was opposition from religious groups because of the belief that illness was a punishment for sin and that God's retribution should not be interfered with. Proponents of the miasma theory objected to vaccination because they believed that illness management was based on a safe and healthy environment. Those supporters of the imbalance in body humours were dependent on, for example blood letting, and herbal and other potions for their income and thus had a pecuniary interest in opposing the introduction of smallpox vaccine.

In contrast, opposition to pertussis in the 1970s and MMR in the 1990s started many years after the germ theory of disease had been accepted when the concept of disease aetiology was integral to healthcare practice. Opposition to these vaccines therefore focused not on disease causation but rather on vaccine adverse events and efficacy. Thus both the pertussis and MMR controversies started with medical professionals publishing a case series of adverse events associated with the respective vaccines and subsequently claiming a causal relationship despite the study design not allowing such an interpretation.

Knowledge about AEFIs following pertussis vaccine had been reported in the medical literature at least from the time of Madsen's use of vaccine in the Faroe Islands in the 1930s [37]: in addition there were several case studies in the 1940s and 1950s drawing attention to the possible association between brain damage and pertussis vaccine $[42,43]$. For MMR prior to 1998, the principal concerns about the vaccine adverse event profile and efficacy related to the mumps component, which had caused an increased frequency of mumps meningitis with the less well attenuated Urabe strain, and the lack of protection associated with the Rubini strain $[60,78]$. The obvious question therefore is what happened in 1973/4 and 1998 that caused the whooping cough and MMR controversies respectively and what does this tell us about the origin and spread of adverse vaccination related publicity?

A review of the incidents would suggest some/ all of the following eight factors might be influential in the origin or spread of vaccine controversies. First they concerned public health programmes administered universally to prevent disease - when delivering such prophylactic programmes one has to be aware of the exhortation "primum non nocere" (above all, do no harm) since the alleged occurrence of damage in healthy recipients is perceived quite differently to adverse events that occur during medical treatment of diseased patients [79]. Furthermore, they were delivered to infants and children - this was also the age group involved in the smallpox controversy two centuries earlier. Infants and children are unable to consent to a procedure and this introduces the element of guilt in parents/ guardians who consented on their behalf if there are adverse events subsequently attributed to it.

Second was the speed with which the information got into the public domain through radio, TV, and newspapers. Wilson's talk and subsequent Lancet paper became headline news within a few months. This was not as rapid as the MMR controversy, which happened within weeks, but in the latter case the lead author called a press conference the day after publication of the Lancet paper to publicise the research, leading to it being the main item on UK news programmes for several days. With any future vaccine controversy such a speedy onset is likely to be accelerated with social media being a key vehicle - healthcare organisations need to be aware of the potential need for a rapid response and be familiar with the use of social media.

Third was the willingness of some individuals within the medical profession to support the adverse publicity, or at least acknowledge the possibility that the research was correct. Even though the numbers of doctors who opposed both the pertussis or MMR vaccines were very small, the publicity they attracted was substantial, thus giving the impression that this was a widely held view in medicine - healthcare organisations need to have well developed mechanisms for addressing vaccine concerns, and it would seem appropriate that they are the same as those used to disseminate 'good news' vaccine stories.

Fourth was the involvement of parents. For pertussis a highly organised, politically aware and articulate parent group came together very early in the controversy, and they were able to bring their own personal/ family experiences to the situation. This both focused the audience's attention on the plight of an individual child, and also made it difficult for a health professional to discuss the issue of possible brain damage and vaccination without appearing to be an unsympathetic, and unfeeling, scientist. There was a similar situation 
with MMR and autism. This is extremely difficult and would involve in any future incident agreeing (where possible) the format of an interview beforehand, clarifying the focus of the interview and the key points that need addressing through to rehearsing the likely questions - note that the different media will vary in how they will conduct an interview. One important caveat is that the health professional being interviewed should be the appropriate person.

Fifth was the involvement and support of the very highly respected MP, Jack Ashley, who himself had a disability (deafness at age 45 years following an operation) and who then had actively campaigned on behalf of disabled people in Parliament receiving broad cross party support for his approach. Ashley presumably was also able to advise how to progress the campaign from a political perspective. Although there were 224 reported debates/ questions about MMR and autism, the Parliamentary opposition seemingly lacked a focal point around which the campaign could be organised $[80,81]$. When addressing the antivaccine movement in any future incident it will be important to think about the source of possible Parliamentary opposition.

Sixth was the lack of data to refute the suggested association between the vaccine and the specified outcome - chronic brain damage and pertussis, and autism, bowel disease and MMR. Current licensing of a vaccine requires preclinical and clinical studies (Phase I, II and III), a successful licensing application and then a Phase IV post licensing study. Pertussis vaccine had not undergone such a comprehensive pre and post licensing programme because it was licensed prior to this being implemented, and for MMR the numbers of children involved and the duration of the longest Phase III follow up study was limited to commonly occurring adverse events: obviously an appropriate balance needs to be struck between the importance of Phase III studies that enable the identification of Adverse Events Following Immunisation (AEFIs) and the earlier implementation of an effective vaccine - whatever the decision, a Phase IV study of appropriate length and rigor needs to be conducted to identify very low frequency AEFIs. These preclinical and clinical data then need to be available at the time the vaccine is implemented in a readily accessible format so that health professionals are in a position to address concerns that arise from parents, guardians, individuals and the media (including social media).

Seventh was the complexity of the arguments involved in universal prophylactic vaccination programmes: thus vaccines protect both by the direct route (generating an adaptive response in the vaccinated individual), and by the indirect route (the concept of herd immunity). Furthermore, different vaccines vary in the balance between direct and indirect protection, and also how effective they are. In addition all vaccines are associated with adverse events be they very common as in local inflammation due to dendritic cell activation with the release of pro-inflammatory cytokines, or extremely rare as in anaphylaxis. Neither protection nor adverse events around pertussis or MMR could easily be discussed in the usual three minutes news slot. This reinforces the critical importance of ensuring that consent to immunisation is truly informed and based on sufficient information and knowledge around the disease (and its associated morbidity and mortality), the vaccine (and basis of vaccination, relevant vaccine type, schedule, efficacy and duration of protection), vaccine contraindications and adverse events, and finally alternate methods of disease management and control. The role of the individual healthcare professional is as essential to this process as is literature from key organisations, for example the $\mathrm{DH}$.
And eighth it is important to recognise that the balance of media reporting does not necessarily reflect the balance of the argument among the involved professionals - by this is meant that equal weighting might be given by the media (and thus the lay community) to those for and against the vaccine although opponents of vaccination might be a very small number, as for example happened with both pertussis and MMR.

\section{Summary}

This paper has described the three vaccine controversies that have occurred in the UK over the past 200 years. It focused on the factors associated with the genesis and progression of each incident looking for similarities and dissimilarities. The oldest incident involved smallpox and took nearly 150 years for major public disquiet to develop - this coincided with the introduction of compulsory vaccination. Whooping cough became a major controversy within a year of the initiating conference presentation although concerns about similar AEFIs had been voiced a number of years earlier. The controversy that developed around MMR started within a few months of the publication of a paper suggesting a possible link between the vaccine and pervasive development. Eight different factors were identified that are believed to be important in explaining how controversies around vaccination start and progress. Suggestions are made about how they may be addressed with any future such incidents.

\section{References}

1. http://digital.nls.uk/indiapapers/vaccination.html.

2. Fitchett JaH DL (2011) Smallpox Vaccination and Opposition by Antivaccination Societies in 19th Century Britain. Historia Medicine 2:21.

3. Timonius E, Woodward J (1714) An Account, or History, of the Procuring the Small Pox by Incision, or Inoculation; As It Has for Some Time Been Practised at Constantinople. Philosophical Transactions 2: 72-82.

4. Boylston A (2012) The Newgate Guinea Pigs. London Historians.

5. Newman H (1722) The Way of Proceeding in the Small Pox Inoculated in New England. Communicated by Henry Newman, Esq; Of the Middle Temple. Philosophical Transactions 32: 33-35.

6. Jewson ND (1974) Medical Knowledge and the Patronage System in 18th Century England. Sociology 8: 369-385.

7. Cohen H (1953) The evolution of the concept of disease. Proceedings of the Royal Society of Medicine 45: 155-160.

8. Delafaye $\mathrm{T}$ (1753) Inoculation an indefensible Practice. Villanova University. The Gobe, Pater Noster-Row, London.

9. White AD (1898) Theological Opposition to Inoculation, Vaccination, and the Use of Aneasthetics. A History of the Warfare of Science with Theology in Christendom. D. Appleton and Company, New York, USA.

10. Ostry A (1994) Theories of disease causation and their impact on public health in 19th century Canada. Can J Public Health 85: 368-369.

11. Carter KC (1991) The development of Pasteur's concept of disease causation and the emergence of specific causes in nineteenth-century medicine. Bull Hist Med 65: 528-548.

12. DeLacy M (1993) The conceptualization of influenza in eighteenthcentury Britain: specificity and contagion. Bull Hist Med 67: 74-118.

13. Anonymous (1721) Post Boy.

14. Parish HJ (1965) A History of Immunization. E \& S Livingstone Ltd, Edinburgh and London.

15. Anonymous (1721) New England Courant.

16. Riedel S (2005) Edward Jenner and the history of smallpox and vaccination. Proc (Bayl Univ Med Cent) 18: 21-25. 
17. Anonymous (1726) Parker's Penny Post.

18. Anonymous (1724) Parker's London News or the Impartial Intelligencer.

19. Kumar VS, Navaratnam V (2013) Neem (Azadirachta indica): prehistory to contemporary medicinal uses to humankind. Asian Pac J Trop Biomed 3: 505-514.

20. Biddle W (1995) A field guide to germs: Allen \& Unwin, 127-34.

21. http://18thcenturyrecipes.wordpress.com/2010/06/17/health-inthe-18th-century/.

22. http://www.jenner.ac.uk/edwardjenner

23. Parish HJ (1968) Victory with Vaccines. E \& S Livingstone Ltd, Edinburgh and London.

24. Anonymous (1853) Imperial Parliament. Daily News.

25. Anonymous (1840) The Chelmsford Chronicle.

26. Wolfe RM, Sharp LK (2002) Anti-vaccinationists past and present. BMJ 325: 430-432.

27. Anonymous. Anti-vaccination Riot in Leicester.

28. Derby Daily Telegraph. 1882.

29. Anonymous (1871) The Standard.

30. Anonymous (1876) Gleanings. Birmingham Daily Post.

31. Anonymous (1871) Compulsory Vaccination Liverpool Mercury.

32. http://www.le.ac.uk/lahs/downloads/ RossPagesfromvolumeXLIIIsm-7.pdf.

33. Williams C (1796) Anti-vaccine cartoon that provided the fore piece to Vaccinae Vindicia. London.

34. Anonymous. Editorial. Manchester Courier and Lancashire General Advertiser.

35. Smart AG (1883) Dust, and disease :two chapters in our life history. MacNiven and Wallace, Edinburgh.

36. Koch R (1880) Investigations into the etiology of traumatic infective diseases. The New Sydenham Society, London.

37. Howson CP, Howe CJ, Fineberg HV (1991) Adverse effects of pertussis and rubella vaccines. National Academy Press, Washington D.C., USA.

38. Madsen $\mathrm{T}$ (1933) Vaccination against whooping cough. JAMA 101:187-188.

39. Kendrick PL (1942) Use of Alum-Treated Pertussis Vaccine, and of Alum-Precipitated Combined Pertussis Vaccine and Diphtheria Toxoid, for Active Immunization. Am j public health nations health 32: 615-626.

40. McFarlan AM, Topley E, Fisher M (1945) Trial of Whooping-cough Vaccine. Br Med J 2: 205-208.

41. Amirthalingam G, Gupta S, Campbell H (2013) Pertussis immunisation and control in England and Wales, 1957 to 2012: a historical review. Euro Surveill 18.

42. Vaccination against whooping-cough (1959) The final report to the Medical Research Council. Br Med J 1: 994-1000.

43. Byers RK, Moll FC (1948) Encephalopathies following prophylactic pertussis vaccine. Pediatrics 1: 437-457.

44. Toomey JA (1949) Reactions to pertussis vaccine. J Am Med Assoc 139 448-450.

45. Hodder SL, Mortimer EA Jr (1992) Epidemiology of pertussis and reactions to pertussis vaccine. Epidemiol Rev 14: 243-267.

46. Sepkowitz S (1996) Perverse reactions to pertussis vaccine by government medical agencies. J Okla State Med Assoc 89: 135-138.

47. Griffith AH (1978) Reactions after pertussis vaccine: a manufacturer's experiences and difficulties since 1964. Br Med J 1: 809-815.

48. Wilson J (1973) Proceedings: Neurological complications of DPT inoculation in infancy. Arch Dis Child 48: 829-830.

49. Wilson J (1975) Letter: Whooping-cough vaccine. Br Med J 4: 459-460.

50. Kulenkampff M, Schwartzman JS, Wilson J (1974) Neurological complications of pertussis inoculation. Arch Dis Child 49: 46-49.

51. Hansard (1974) Immunisation. Hansard.

52. Lee-Potter L (1974) The questions we Must ask. Daily Mail.
53. Stewart GT (1976) Letter: Immunisation against whooping cough. Br Med J 1: 283.

54. Stewart GT (1976) Letter: Immunisation against whooping cough. Br Med J 1: 93-94.

55. Bassili WR, Stewart GT (1976) Epidemiological evaluation of immunisation and other factors in the control of whooping-cough. Lancet 1: 471-474.

56. Stewart GT (1977) Vaccination against whooping-cough. Efficacy versus risks. Lancet 1: 234-237.

57. Stewart GT (1979) Toxicity of pertussis vaccine: frequency and probability of reactions. J Epidemiol Community Health 33: 150-156.

58. Dick G (1975) Letter: Whooping-cough vaccine. Br Med J 4: 161.

59. http://www.jabs.org.uk/pages/fox.asp.

60. Baker JP (2003) The pertussis vaccine controversy in Great Britain, 1974-1986. Vaccine 21: 4003-4010.

61. Miller D, Madge N, Diamond J, Wadsworth J, Ross E (1993) Pertussis immunisation and serious acute neurological illnesses in children. BMJ 307: 1171-1176.

62. Miller DC, Wadsworth MJ, Ross EM (1989) Pertussis vaccine and severe acute neurological illnesses. Response to a recent review by members of the NCES team. Vaccine 7: 487-489.

63. Miller D, Wadsworth J, Diamond J, Ross E (1985) Pertussis vaccine and whooping cough as risk factors in acute neurological illness and death in young children. Dev Biol Stand 61: 389-394.

64. Stephenson JB (1988) A neurologist looks at neurological disease temporally related to DTP immunization. Tokai J Exp Clin Med 13 Suppl: 157-164.

65. Griffith AH (1989) Permanent brain damage and pertussis vaccination: is the end of the saga in sight? Vaccine 7: 199-210.

66. Dyer C (1988) Judge "not satisfied" that whooping cough vaccine causes permanent brain damage. Br Med J (Clin Res Ed) 296: 1189-1190.

67. Wakefield AJ, Murch SH, Anthony A, Linnell J, Casson DM, et al. RETRACTED: Ileal-lymphoid-nodular hyperplasia, non-specific colitis, and pervasive developmental disorder in children. The Lancet 351: 637-641.

68. Wakefield AJ, Murch SH, Anthony A, Linnell J, Casson DM, et al. (1998) Ileal-lymphoid-nodular hyperplasia, non-specific colitis, and pervasive developmental disorder in children. Lancet 351: 637-641.

69. Peltola H, Patja A, Leinikki P, Valle M, Davidkin I, et al. (1998) No evidence for measles, mumps, and rubella vaccine-associated inflammatory bowel disease or autism in a 14-year prospective study. Lancet 351: 1327-1328.

70. Wakefield AJ, Montgomery SM (2000) Measles, mumps, rubella vaccine: through a glass, darkly. Adverse Drug React Toxicol Rev 19: 265-283.

71. Uhlmann V, Martin CM, Sheils O, Pilkington L, Silva I, et al. (2002) Potential viral pathogenic mechanism for new variant inflammatory bowel disease. Mol Pathol 55: 84-90.

72. Madsen KM, Hviid A, Vestergaard M, Schendel D, Wohlfahrt J, et al. (2002) A population-based study of measles, mumps, and rubella vaccination and autism. N Engl J Med 347: 1477-1482.

73. Patja A, Davidkin I, Kurki T, Kallio MJ, Valle M, et al. (2000) Serious adverse events after measles-mumps-rubella vaccination during a fourteen-year prospective follow-up. Pediatr Infect Dis J 19: 1127-34.

74. Honda H, Shimizu Y, Rutter M (2005) No effect of MMR withdrawal on the incidence of autism: a total population study. J Child Psychol Psychiatry 46: 572-579.

75. Taylor B, Miller E, Farrington CP, Petropoulos MC, Favot-Mayaud I, et al. (1999) Autism and measles, mumps, and rubella vaccine: no epidemiological evidence for a causal association. Lancet 353: 2026-2029.

76. Goodman NW (2007) MMR scare stories: Some things are just too attractive to the media. BMJ 335: 222.

77. Hussain H, Omer SB, Manganello JA, Kromm EE, Carter TC, et al. (2011) Immunization safety in US print media, 1995-2005. Pediatrics 127 Suppl 1: S100-106. 
Citation: Baxter D (2014) Opposition to Vaccination and Immunisation the UK Experience - from Smallpox to MMR. J Vaccines Vaccin 5: 254. doi:/10.4172/2157-7560.1000254

Page 8 of 8

78. Zetterström R (2004) Flawed reports of immunization complications: consequences for child health. Acta Paediatr 93: 1140-1143.

79. Dayan GH, Rubin S (2008) Mumps outbreaks in vaccinated populations: are available mumps vaccines effective enough to prevent outbreaks? Clinical infectious diseases : an official publication of the Infectious Diseases Society of America 47: 1458-1467.
80. Smith CM (2005) Origin and uses of primum non nocere--above all, do no harm! J Clin Pharmacol 45: 371-377.

81. http://www.parliament.uk/search/results/?q=MMR+and+Autism. 